\title{
Multiscale Computational Strategies for Heterogeneous Materials with Defects: Coupling Modeling with Experiments and Uncertainty Quantification
}

\author{
SOMNATH GHOSH (1) ${ }^{1,3}$ and DAVID MCDOWELL ${ }^{2}$ \\ 1.-Johns Hopkins University, Baltimore, MD, USA. 2.-Georgia Institute of Technology, Atlanta, \\ GA, USA. 3.-e-mail: sghosh20@jhu.edu
}

The demand for effective and robust computational platforms in physics-based multi-scale modeling is on the rise to realize improved structurematerials systems. These computational tools have the potential to unravel important underpinnings of microstructure-property relationships, enabling better materials and process design. Robustness of computational tools is determined by the level of verification and validation of the models and codes. This requires synergy between experiments and modeling in terms of incorporation of physics, calibration, and validation. In this context, it has become increasingly evident that uncertainty quantification (UQ) is a key "leg of a stool" that supports the fusion of experiments with computational modeling. Moreover, with the accelerated application of data science methods to enhance model-experiment fusion, there is a clear preference for methods that can quantify the uncertainty of the interpretation of information, whether expressed in terms of random variability, model forms and/or parameters, experimental uncertainty, lack of information, or improvement of understanding of phenomena via experiments.

The materials community has focused much attention in the last decade on digital 3D microstructures and the associated uncertainty of measurements and methods used to represent material structure. In contrast, this special topic focuses more on modeling and experiments that facilitate understanding necessary to design or manipulate material structures to achieve desired performance targets, or to quantify variability of responses for a given material. The need to quantify uncertainty and track uncertainty propagation (UQUP) is particularly compelling for understanding and modeling of the responses of materials with a hierarchy of scales of structure. Experiments are conducted at various levels of spatial and temporal resolution to measure the evolution and impact of defects, while multiscale models are constructed to express the interplay of these scales in conferring response at the scale of applications. This is true for atomic-scale structures, for interfaces such as grain or phase boundaries (including their networks), for other defect structures (e.g., voids, dislocations), and for the morphology of microstructure at scales ranging from hundreds of nanometers to tens of microns. The ability to tailor material structure and therefore responses is one of the primary aims of integrated computational materials engineering and the Materials Genome Initiative. For such multiscale material systems, widely prevalent in applications, UQ and forward propagation through chained systems of models are critically important in providing decision support in materials selection and design. Moreover, inverse UQ that considers consistency with higher-scale experiments or models is vital to establishing a self-consistent hierarchical multiscale model framework.

This special topic attempts to provide the reader with an overview of some of the key challenges pursuant to multiscale modeling of complex engineering material systems. UQUP is an underpinning cornerstone necessary to address these challenges, including:

- Combined use of computational simulation and experiments conducted at various levels of spatiotemporal resolution and fidelity to calibrate model parameters

- Informing the construction of reliable hierarchical multiscale models that combine modeling and experimental information from the "bottom-up" as well as the "top-down" and to assess confidence in understanding of key phenomena and mechanisms in material responses

- Providing support for quantifying uncertainty of the structure or form of models, as well as methods for linking information exchange be- 
tween levels of hierarchical models or between levels in concurrent multiscale models

- Uncertainty in experiments, including both measurement error and approximations in algorithms used to interpret the measurements to understand material process-structure and structure-property relationships

This special topic is a collection of 13 papers that explore and develop physics-based multiscale computational methods with a focus on experimental calibration and validation, including uncertainty quantification. The papers are organized into four groups that may be classified according to (1) multiscale modeling of deformation and failure across a range of microstructures, (2) UQ in modeling materials with microstructure, (3) homogenization and reduced order modeling with uncertainty quantification, and (4) effective coupling of experiments with modeling. A list of the paper titles are given at the end of this section. A diverse range of state-of-the-art research topics are addressed by leading experts in the respective fields.

Four papers constitute the first group of multiscale modeling. They include experiments and density functional theory, and phase field modeling of deformation and failure in ceramic composites; discrete dislocation plasticity modeling of microstructure dependence of dislocation configurational energy; crystal plasticity modeling of ratcheting in polycrystalline metals; and a phase-fieldinformed crystal plasticity model for predicting the mechanical response of superalloys.

The second group of three papers concern UQ in microstructure-sensitive modeling. These papers address understanding the effect of distributions of attributes of microstructure on crystal plasticity model parameters and resulting stochasticity of mechanical response; UQ in metal additive manufacturing processes using physics-informed datadriven modeling with experimental validation; and a description of UQ tools on the nanoHUB.

The third group consists of two papers on homogenization and reduced order modeling with UQ. The first paper entails a Gaussian process-driven adaptive sampling for reduced order modeling of texture effects in polycrystalline Ti alloys, while the second paper discusses parametric homogenization-based constitutive and crack nucleation models for $\mathrm{Ti}$ alloys with UQ.

The final group of four papers explores effective methods to couple experiments with modeling for purposes of calibrating and validating models. These include a paper on the optimal design of experiments for the extraction of single-crystal material properties from spherical indentation measurements; use of machine learning to extract microstructure-sensitive evolution of a crack in a polycrystalline alloy; use of laboratory diffraction contrast tomography (LabDCT) for high-reliability grain mapping; and an integrated experimental modeling approach to understanding and quantifying dislocation-defect interactions during hot working of fcc alloys.

We trust this special issue will provide the reader an improved perspective and understanding of the key challenges, along with some potential approaches to construct useful multiscale models that couple with experiments via formal UQ. Model-experiment fusion with UQUP is a rapidly developing field which has only recently attracted the focus it deserves.

The following list summarizes the papers being published under the topic of "Multiscale Computational Strategies for Heterogeneous Materials with Defects: Coupling Modeling with Experiments and Uncertainty Quantification". To download any of the papers, follow the URL http://link.springer.com/ journal/11837/71/8/page/ 1 to the table of contents page for the August 2019 issue (vol. 71, no. 8).

Group I. Microstructural and Multi-scale Modeling of Deformation and Failure

1. "Deformation and Failure Mechanics of Boron Carbide-Titanium Diboride Composites at Multiple Scales" by J.D. Clayton, W.S. Rubink, V. Ageh, D. Choudhuri, R. Recuero Chen, J. Du, and T.W. Scharf

2. "Effects of Grain Size, Orientation and Source Density Study of Dislocation Configurational Energy Density" by Zebang Zheng and Fionn P.E. Dunne

3. "Local Ratcheting Phenomena in the Cyclic Behavior of Polycrystalline Tantalum" by Damien Colas, Eric Finot, Sylvain Flouriot, Samuel Forest, Matthieu Mazière, and Thomas Paris

4. "Finite-Element Crystal Plasticity On PhaseField Microstructures: Predicting Mechanical Response Variations in Ni-Based Single Crystal Superalloys" by Jean-Briac le Graverend and Rajendran Harikrishnan

Group II. Uncertainty Quantification in Microstructural Modeling

5. "Uncertainty Quantification in the Mechanical Response of Crystal Plasticity Simulations" by Ritwik Bandyopadhyay, Veerappan Prithivirajan, and Michael D. Sangid

6. "Uncertainty Quantification in Metallic Additive Manufacturing Through Physics-Informed Data-Driven Modeling” by Zhuo Wang, Pengwei Liu, Yanzhou Ji, Sankaran Mahadevan, Mark F. Horstemeyer, Zhen Hu, Lei Chen, and LongQing Chen

7. "Online Tools for Uncertainty Quantification in nanoHUB" by Saaketh Desai, Martin Hunt, and Alejandro Strachan 
Group III. Homogenization and Reduced Order Modeling with Uncertainty Quantification

8. "Gaussian-Process-Driven Adaptive Sampling for Reduced Order Modeling of Texture Effects in Polycrystalline Alpha-Ti” by Aaron E. Tallman, Krzysztof S. Stopka, Laura P. Swiler, Yan Wang, Surya R. Kalidindi, and David L. McDowell

9. "Parametrically Homogenized Constitutive Models (PHCMs) for Multi-Scale Predictions of Fatigue Crack Nucleation in Titanium Alloys" by Deniz Ozturk, Shravan Kotha, Adam L. Pilchak, and Somnath Ghosh

\section{Group IV. Coupling Experiments with Modeling}

10. "Bayesian Sequential Design of Experiments for the Extraction of Single-Crystal Material Properties from Spherical Indentation Mea- surements on Polycrystalline Samples" by Andrew R. Castillo, V. Roshan Joseph, and Surya R. Kalidindi

11. "Predicting Microstructure-Sensitive FatigueCrack Path in 3D Using a Machine Learning Framework" by Kyle Pierson, Aowabin Rahman, and Ashley D. Spear

12. "A Forward Modeling Approach to High Reliability Grain Mapping by Laboratory Diffraction Contrast Tomography (LabDCT)" by Sridhar Niverty, Jun Sun, Jason Williams, Florian Bachmann, Nicolas Gueninchault, Erik Lauridsen, and Nikhilesh Chawla

13. "Integrated Experimental-Modeling Strategy to Understand Dislocation-Defect Interactions During Hot Working of Face-Centered Cubic Alloys" by B. Aashranth, Santosh Kumar, Dipti Samantaray, and Utpal Borah 